\title{
Notes on reflection and refraction of evanescent plane waves on the boundary between gainy/lossy media
}

\author{
Jozefa Červeňová, Rastislav Dosoudil, \\ Jaroslav Franek, L’ubomír Šumichrast ${ }^{1}$
}

\begin{abstract}
Reflection and transmission (refraction) of a homogeneous plane wave at the planar boundary of two dielectric media is a well known phenomenon commonly treated in nearly all standard textbooks. Here the analysis of reflection and refraction of evanescent plane waves on the planar boundary between various combinations of lossy, gainy and lossless media is performed. It is shown that by the appropriate choice of the profile of evanescence various effects can take place.
\end{abstract}

K e y w ord s: reflection, refraction, evanescent plane waves, gainy/lossy media

\section{Introduction}

The reflection and refraction of homogeneous plane waves on the planar boundary between two lossless dielectrics is a well known phenomenon presented in nearly all textbooks on electromagnetism, eg [1-3]. The evanescent waves are treated therein usually only in context of the total internal reflection of homogeneous plane waves on the boundary between two lossless dielectrics. Here the phenomena occurring within the framework of the evanescent plane waves propagating in the lossy, gainy, or lossless media and reflected/refracted on the planar boundary between them, are thoroughly analysed.

\section{Propagation of an evanescent plane wave in lossy, or gainy medium}

Consider an infinite non homogeneous harmonic plane wave of angular frequency $\omega$ with electric and magnetic field intensity vectors $\hat{\mathbf{E}}(\boldsymbol{r}, t)=\operatorname{Re}\{\mathbf{E}(\boldsymbol{r}, t)\}$ and $\hat{\mathbf{H}}(\boldsymbol{r}, t)=\operatorname{Re}\{\mathbf{H}(\boldsymbol{r}, t)\}$ in complex representation of the form

$$
\begin{aligned}
& \mathbf{E}(\boldsymbol{r}, t)=\mathbf{E}(\boldsymbol{r}) \exp (j \omega t), \\
& \mathbf{H}(\boldsymbol{r}, t)=\mathbf{H}(\boldsymbol{r}) \exp (j \omega t),
\end{aligned}
$$

propagating in lossy (attenuating), or gainy (amplifying) medium, with the propagation constant

$$
\gamma=\sqrt{j \omega \mu(j \omega \varepsilon \pm \sigma)}=j \tilde{\beta} \pm \tilde{\alpha}
$$

where upper plus sign holds for lossy medium and lower minus sign for gainy medium, respectively, and $\sigma$ and $\alpha$ is considered always having non negative value.

Throughout the whole paper when double sign is used then the uper sign is used for lossy and the lower for gainy medium. For our purposes it is more convenient to use $\gamma$ in the form

$$
\gamma=\sqrt{-\beta^{2} \pm j \alpha^{2}}
$$

where $\beta=\omega \sqrt{\mu \varepsilon}$ is the same phase constant as in the case of an ideal lossless dielectrics $(\sigma=0)$ and $\alpha=$ $\sqrt{\omega \mu \sigma}$. The standardly used phase constant $\tilde{\beta}$ and the attenuation/amplification constant $\tilde{\alpha}$ in (2) are related to $\alpha$ and $\beta$ by

$$
\begin{aligned}
& \tilde{\beta}^{2}=\frac{1}{2}\left\{\beta^{2}+\sqrt{\beta^{4}+\alpha^{4}}\right\}, \\
& \tilde{\alpha}^{2}=\frac{1}{2}\left\{-\beta^{2}+\sqrt{\beta^{4}+\alpha^{4}}\right\},
\end{aligned}
$$

or vice versa by

$$
\beta^{2}=\tilde{\beta}^{2}-\tilde{\alpha}^{2}, \quad \alpha^{2}=2 \tilde{\beta} \tilde{\alpha}
$$

In what follows, for the sake of brevity, we shall consider only a two dimensional TE case, where

$$
\mathbf{E}(\boldsymbol{r})=\mathbf{u}_{y} E(x, z), \quad \mathbf{H}(\boldsymbol{r})=\mathbf{u}_{x} H_{x}(x, z)+\mathbf{u}_{z} H_{z}(x, z) .
$$

Generally one has first to solve the wave equation for $E(x, z)$

$$
\frac{\partial^{2} E(x, z)}{\partial x^{2}}+\frac{\partial^{2} E(x, z)}{\partial z^{2}}-\gamma^{2} E(x, z)=0
$$

and then, subsequently, determine $\mathbf{H}(x, z)$ from the Maxwell equation

$$
\mathbf{H}(x, z)=\frac{j}{\omega \mu}\left[-\frac{\partial E(x, z)}{\partial z} \mathbf{u}_{x}+\frac{\partial E(x, z)}{\partial x} \mathbf{u}_{z}\right] .
$$

The solution of (5) in form of an evanescent wave propagating in $z$ direction is given by the electric intensity vector

$$
\mathbf{E}(x, z)=\boldsymbol{u}_{y} E \exp (\varkappa x) \exp (\psi z) \exp (-j \kappa z),
$$

\footnotetext{
${ }^{1}$ Slovak University of Technology, Institute of Electrical Engineering, Ilkovičova 3, SK-81219 Bratislava, Slovakia, lubomir.sumichrast@stuba.sk
} 


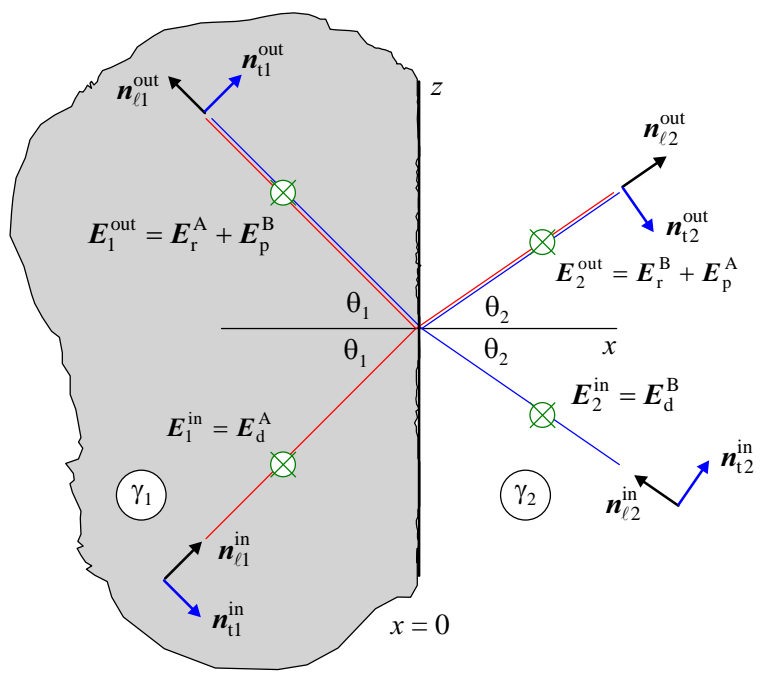

Fig. 1. Schematics of the wave propagation along the boundary consisting of two triples of incident, reflected and transmitted wave

where $\kappa$ is the wave number of the propagating wave, $\psi$ the attenuation/amplification factor in direction of propagation with $\psi>0$ for gainy medium, and $\psi<0$ for lossy medium respectively, and $\varkappa$ is the measure of transversal evanescence of the amplitude of propagating wave, either with $\varkappa>0$ - here called negative evanescence (decay of the wave amplitude in the negative $x$ direction), or with $\varkappa<0$ - here called positive evanescence (decay of the wave amplitude in the positive $x$ direction).

For $\varkappa=0,(7)$ represents a homogeneous plane wave. The wavenumber $\kappa$ has always a positive value.

The second solution of (5) is a back propagating evanescent wave, ie propagating in minus $z$ direction, simply given as (7) but with all signs reversed.

Substitution of (7) into the wave equation (5) leads to $\varkappa^{2}+\left(-j \kappa+\psi^{2}\right)+\beta^{2} \mp j \alpha^{2}=0$ and results in two equations for its real and imaginary part

$$
\begin{aligned}
& \kappa^{2}=\psi^{2}+\varkappa^{2}+\beta^{2}, \\
& 2 \kappa \psi= \pm \alpha^{2},
\end{aligned}
$$

yielding thus the wavenumber $\kappa$ and attenuation factor $\psi$ of the propagating evanescent wave as a function of the media parameters and $\alpha$ and the evanescence factor of the wave $\varkappa$, ie

$$
\begin{aligned}
& \kappa^{2}=\frac{1}{2}\left\{\left(\beta^{2}+\varkappa^{2}\right)+\sqrt{\left(\beta^{2}+\varkappa^{2}\right)^{2}+\alpha^{4}}\right\}>\tilde{\beta}^{2} \\
& \psi^{2}=\frac{1}{2}\left\{-\left(\beta^{2}+\varkappa^{2}\right)+\sqrt{\left(\beta^{2}+\varkappa^{2}\right)^{2}+\alpha^{4}}\right\}<\tilde{\alpha}^{2} .
\end{aligned}
$$

The phase constant $\kappa$ is always equal to positive root of (9), while $\psi$ in accordance with $\psi=\mp \alpha^{2} / 2 \kappa$ is negative for lossy medium and positive for gainy medium.

It is clearly seen that the wavenumber $\kappa$ is always larger than the phase constant $\tilde{\beta}$ ie the phase velocity of such an evanescent plane wave, sometimes called "slow wave", is always smaller than that of a homogeneous plane wave (the case $\varkappa=0$ ) propagating in the same medium. The attenuation/amplification factor $\psi$ is always smaller than the attenuation/amplification constant $\tilde{\alpha}$ of homogeneous plane wave. The result is independent of whether the evanescence is positive, or negative, or whether the medium is lossy or gainy. For a homogeneous wave, $i e$ for $\varkappa=0$ as seen from (9), (10), $\kappa=\tilde{\beta}$ and $\psi=\tilde{\alpha}$.

In case of a lossless medium $\kappa=\sqrt{\beta^{2}+\varkappa^{2}}, \psi=0$ and

$$
\mathbf{E}(x, z)=\boldsymbol{u}_{y} E \exp (\varkappa x) \exp (-j \kappa z),
$$

$i e$ in a lossless medium an undamped "slow wave" propagates along the $z$ axis with evanescent profile along the $x$ axis.

The magnetic intensity vector $\mathbf{H}(x, z)$ pertaining to (7) is given by

$$
\begin{aligned}
\mathbf{H}(x, z)=\frac{E}{\omega \mu}[-(\kappa+ & \left.j \psi) \mathbf{u}_{x}+j \varkappa \mathbf{u}_{z}\right] \\
& \times \exp (\varkappa x) \exp \{-j(\kappa+j \psi) z\}
\end{aligned}
$$

and the power flow density (Poynting vector) $\mathbf{S}(x, z)=$ $\frac{1}{2} \operatorname{Re}\{\mathbf{E} \times \mathbf{H} *\}$ reads

$$
\mathbf{S}(x, z)=\frac{\kappa|E|^{2}}{2 \omega \mu} \mathbf{u}_{z} \exp (2 \varkappa x) \exp (2 \psi z)
$$

\section{Oblique propagation of an evanescent plane wave}

Let us now consider oblique propagation of an evanescent wave in direction of the longitudinal unit vector $\boldsymbol{n}_{\ell}$ with the evanescent profile in direction of the transversal unit vector $\boldsymbol{n}_{t}=\boldsymbol{u}_{y} \times \boldsymbol{n}_{\ell}$ where

$$
\begin{aligned}
& \mathbf{n}_{\ell}=\boldsymbol{u}_{z} \sin \theta+\mathbf{u}_{x} \cos \theta, \\
& \mathbf{n}_{t}=-\boldsymbol{u}_{z} \cos \theta+\boldsymbol{u}_{x} \sin \theta,
\end{aligned}
$$

$i e$ the unit vector $\boldsymbol{n}_{\ell}$ spans the angle $\theta$ with the positive $x$ axis (as $e g$ for the wave with $\mathbf{E}_{1}^{\text {in }}$ the angle $\theta_{1}$ or for $\mathbf{E}_{2}^{\text {out }}$ the angle $\theta_{2}$ in Fig. 1). Then (7) is transformed into

$$
\begin{array}{r}
\mathbf{E}(\xi, \zeta)=\boldsymbol{u}_{y} E \exp \left(\varkappa \mathbf{n}_{t} \cdot \boldsymbol{r}\right) \exp \left\{-j(\kappa+j \psi) \mathbf{n}_{\ell} \cdot \boldsymbol{r}\right\} \\
=\mathbf{u}_{y} E \exp (\varkappa \xi) \exp \{-j(\kappa+j \psi) \zeta\}
\end{array}
$$

where $\zeta$ and $\xi$ are the longitudinal and transversal variables, ie

$$
\begin{aligned}
& \zeta=\mathbf{n}_{\ell} \cdot \boldsymbol{r}=z \sin \theta+x \cos \theta, \\
& \xi=\mathbf{n}_{t} \cdot \boldsymbol{r}=-z \cos \theta+x \sin \theta .
\end{aligned}
$$

The $\mathbf{H}(\xi, \zeta)$ pertaining to (15) is obtained from the formula analogous to (6) in the form

$$
\begin{aligned}
\mathbf{H}(\xi, \zeta)=\frac{E}{\omega \mu}[-(\kappa+ & \left.j \psi) \mathbf{n}_{t}+j \varkappa \mathbf{n}_{\ell}\right] \\
& \times \exp (\varkappa \xi) \exp \{-j(\kappa+j \psi) \zeta\},
\end{aligned}
$$


Table 1.

\begin{tabular}{lccc}
\hline evanescence & medium & $\exp (g x), g=\chi+b$ & $\exp (h z), h=\vartheta-a$ \\
\hline positive, $a, b<0$ & lossy, $\vartheta, \chi<0$ & $g<0$ & $h<0$ if $|\vartheta|>|a|, h>0$, if $|\vartheta|<|a|$ \\
negative, $a, b>0$ & lossy, $\vartheta, \chi<0$ & $g>0$ if $|\chi|<b, g<0$ if $|\chi|>b$ & $h<0$ \\
negative, $a, b>0$ & gainy, $\vartheta, \chi>0$ & $g>0$ & $h<0$ if $\vartheta<a, h>0$, if $\vartheta>a$ \\
positive, $a, b<0$ & gainy, $\vartheta, \chi>0$ & $g>0$ if $\chi>|b|, g<0$ if $\chi<|b|$ & $h>0$ \\
\hline
\end{tabular}

with the Poynting vector, in analogy to (13) equal to

$$
\mathbf{S}(\xi, \zeta)=\frac{\kappa|E|^{2}}{2 \omega \mu} \mathbf{n}_{\ell} \exp (2 \varkappa \xi) \exp (2 \psi \zeta)
$$

After having introduced

$$
\begin{aligned}
& a=\varkappa \cos \theta, \quad b=\varkappa \sin \theta, \\
& \vartheta=\psi \sin \theta, \quad \chi=\psi \cos \theta, \\
& k=\kappa \sin \theta, \quad q=\kappa \cos \theta,
\end{aligned}
$$

one obtains the exponential terms in $(15)$, in the $(x, z)$ coordinate system, in the form

$$
\exp \{(\chi+b) x\} \exp \{(\vartheta-a) z\} \exp \{-j(q x+k z)\}
$$

with $a, b<0$, for the positive evanescence and $a, b>0$ for the negative evanescence, as well as with $\vartheta, \chi>0$ for gainy media and $\vartheta, \chi<0$ for lossy media.

These results are summarised in Tab. 1, where

$$
g=\chi+b, \quad h=\vartheta-a,
$$

with the following relations

$$
\begin{aligned}
& g^{2}+h^{2}=\varkappa^{2}+\psi^{2}, \\
& \varkappa=g \sin \theta-h \cos \theta, \\
& \psi=g \cos \theta+h \sin \theta .
\end{aligned}
$$

The electric intensity vector $(15)$ in $(x, z)$ coordinate system reads

$$
\mathbf{E}(x, y)=\mathbf{u}_{y} E \exp (g x+h z) \exp \{-j(q x+k z)\} .
$$

The magnetic intensity vector, and the Poynting vector pertaining to (23) read

$$
\begin{aligned}
& \mathbf{H}(x, z)=\frac{E}{\omega \mu}[\left.-(k+j h) \mathbf{u}_{x}+(q+j g) \mathbf{u}_{z}\right] \\
& \times \exp (g x+h z) \exp \{-j(q x+k z)\}, \\
& \boldsymbol{S}(x, z)=\frac{E^{2}}{2 \omega \mu}\left(k \mathbf{u}_{z}+q \mathbf{u}_{x}\right) \exp (2 g x) \exp (2 h z) .
\end{aligned}
$$

As seen from Tab. 1, for the combination of positive evanescence wave propagating in lossy media, or negative evanescence wave propagating in gainy media, the wave amplitude along the $x$ axis is always decreasing for positive/lossy, and increasing for negative/gainy combination, while along the $z$ axis it may be either decreasing, remaining constant, or increasing, depending on the value of $h=\vartheta-a$.

For the combination of negative evanescence wave propagating in lossy media, or positive evanescence wave propagating in gainy media, one obtains the wave amplitude along the $z$ axis always decreasing for negative/lossy, and increasing for positive/gainy combination, while along the $x$ axis it may again be either decreasing, remaining constant, or increasing, depending on the value of $g=b+\chi$.

The case $h=0$ occurs for $\varkappa=\psi \tan \theta$, larger $\varkappa$ yields $h<0$ for lossy/positive combination, $\varkappa$ smaller than $\psi \tan \theta$ yields $h>0$ for lossy/positive combination and vice versa for gainy/negative one. Similarly the case $g=0$ occurs for $f=-\psi \cot \theta$ etc.

Thus, by an appropriate choice of magnitude of evanescence $\varkappa$ one can adjust positive, negative or zero exponential dependence of the wave amplitude in either $z$ or $x$ direction for both, either lossy, or gainy media.

If the $x$ component of the unit vector $\boldsymbol{n}_{\ell}$ is directed in minus $x$ direction, $i e$ if it spans the angle $\pi-\theta$ with the positive $x$ axis (as $e g$ for the wave with $\mathbf{E}_{1}^{\text {out }}$ or $\mathbf{E}_{2}^{\text {in }}$ in Fig. 1), then

$$
\begin{gathered}
\mathbf{n}_{\ell}=\mathbf{u}_{z} \sin \theta-\mathbf{u}_{x} \cos \theta, \\
\mathbf{n}_{t}=\mathbf{u}_{z} \cos \theta+\boldsymbol{u}_{x} \sin \theta, \\
\zeta=\mathbf{n}_{\ell} \cdot \boldsymbol{r}=z \sin \theta-x \cos \theta, \\
\xi=\mathbf{n}_{t} \cdot \boldsymbol{r}=z \cos \theta+x \sin \theta,
\end{gathered}
$$

and instead of (20) one obtains

$$
\exp \{(b-\chi) x\} \exp \{(\vartheta+a) z\} \exp \{j(q x-k z)\} .
$$

The direction of propagation along the $x$ axis is reversed, therefore the sign at $\chi$ in (27) as compared to (20) is reversed too, and, similarly, also the direction of evanescence is reversed with respect to $z$ axis (see Fig. 1), therefore the sign at $a$ is reversed too. The possible combinations are indicated in Tab. 2 for

$$
s=\chi-b, \quad p=\vartheta+a,
$$

with the following relations

$$
\begin{aligned}
& s^{2}+p^{2}=\varkappa^{2}+\psi^{2}, \\
& \varkappa=-s \sin \theta+p \cos \theta, \\
& \psi=s \cos \theta+p \sin \theta .
\end{aligned}
$$


Table 2.

\begin{tabular}{lccc}
\hline evanescence & medium & $\exp (-s x), s=\chi-b$ & $\exp (p z), p=\vartheta+a$ \\
\hline negative, $a, b>0$ & lossy, $\vartheta, \chi<0$ & $s<0$ & $p>0$ if $|\vartheta|<a, p<0$, if $|\vartheta|>a$ \\
positive, $a, b<0$ & lossy, $\vartheta, \chi<0$ & $s>0$, if $|\chi|<|b|, s<0$ if $|\chi|>|b|$ & $p>0$ \\
positive, $a, b<0$ & gainy, $\vartheta, \chi>0$ & $s>0$ & $p>0$ if $\vartheta>|a|, p<0$ if $\vartheta<|a|$ \\
negative, $a, b>0$ & gainy, $\vartheta, \chi>0$ & $s>0$, if $\chi>b, s<0$ if $\chi<b$ & $p>0$ \\
\hline
\end{tabular}

The electric intensity vector $(15)$ in $(x, z)$ coordinate system reads

$$
\mathbf{E}(x, y)=\boldsymbol{u}_{y} E \exp (-s x+p z) \exp \{-j(-q x+k z)\}
$$

where $s>0$ means the wave amplified in minus $x$ direction, $i e$ in the $x$ component of propagation direction $\boldsymbol{n}_{\ell}$.

The magnetic intensity vector $\mathbf{H}(x, z)$ and the Poynting vector $\mathbf{S}(x, z)$ pertaining to $(23)$ read

$$
\begin{aligned}
\mathbf{H}(x, z)=\frac{E}{\omega \mu}\left[-(k+j p) \mathbf{u}_{x}-(q+j s) \mathbf{u}_{z}\right] & \\
& \times \exp (-s x+p z) \exp \{-j(-q x+k z)\}, \\
\mathbf{S}(x, z)= & \frac{E^{2}}{2 \omega \mu}\left[k \boldsymbol{u}_{z}-q \mathbf{u}_{x}\right] \exp (-2 s x) \exp (2 p z) .
\end{aligned}
$$

Similar findings as for Tab. 1 are valid for Tab. 2 too. As seen from Tab. 2, for the combination of positive evanescence wave propagating in lossy media, or negative evanescence wave propagating in gainy media, the wave amplitude along the $z$ axis is always decreasing for positive/lossy, and increasing for negative/gainy combination, while along the $x$ axis it may be either decreasing, remaining constant, or increasing, depending on the value of $s=\chi-b$.

For the combination of negative evanescence wave propagating in lossy media, or positive evanescence wave propagating in gainy media, one obtains the wave amplitude along the minus $x$ axis always decreasing for negative/lossy, and increasing for positive/gainy combination, while along the $z$ axis it may again be either decreasing, remaining constant, or increasing, depending on the value of $p=\vartheta+a$.

Thus, the waves propagating in mutually inverse $x$ direction (as $e g$ the waves $\mathbf{E}_{1}^{\text {in }}$ and $\mathbf{E}_{2}^{\text {out }}$ versus $\mathbf{E}_{1}^{\text {out }}$ and $\mathbf{E}_{2}^{\text {in }}$ in Fig. 1) possess the inverse evanescence, e.g. positive/lossy combination in Tab. 1 corresponds to negative/lossy combination in Tab. 2, similarly negative/gainy to positive/gainy, negative/lossy to positive/lossy and positive/gainy to negative/gainy, both with $g=s$ and $h=p$ Thus, for $e g$ the overall field $\mathbf{E}_{1}=\mathbf{E}_{1}^{\text {in }}+\mathbf{E}_{2}^{\text {out }}$ it holds

$$
\begin{aligned}
\mathbf{E}_{1}(x, y)=\mathbf{u}_{y}\left\{E_{1}^{\text {in }} \exp [(g-j q) x]+\right. & \left.E_{1}^{\text {out }} \exp [(j q-g) x]\right\} \\
& \times \exp \{(-j k+h) z\} .
\end{aligned}
$$

Inserting (23), or (30) with $g=s h=p$ into the wave equation (5) yields $g^{2}-q^{2}-2 j q g+h^{2}-k^{2}-2 j k h+\beta^{2} \mp$ $j \alpha^{2}=0$ with the real part

$$
g^{2}+h^{2}-q^{2}-k^{2}+\beta^{2}=0
$$

equivalent to (8), since $g^{2}+h^{2}=\kappa^{2}+\psi^{2}$ and $q^{2}+k^{2}=\kappa^{2}$ as well as with the imaginary part equal to

$$
k h+q g=\mp \alpha^{2} / 2 \text {. }
$$

equivalent to $\kappa \psi=\mp \alpha^{2} / 2$ in (8).

Solving (33) and (34) for $q$ and $g$ expressed by $k$ and $h$ yields

$g^{2}=\frac{\sqrt{\left(\beta^{2}-k^{2}+h^{2}\right)^{2}+\left(\alpha^{2} \pm 2 k h\right)^{2}}+\left(\beta^{2}-k^{2}+h^{2}\right)}{2}$,

$g^{2}=\frac{\sqrt{\left(\beta^{2}-k^{2}+h^{2}\right)^{2}+\left(\alpha^{2} \pm 2 k h\right)^{2}}-\left(\beta^{2}-k^{2}+h^{2}\right)}{2}$,

or vice versa $k$ and $h$ expressed by $q$ and $g$ yields

$k^{2}=\frac{\sqrt{\left(\beta^{2}-q^{2}+g^{2}\right)^{2}+\left(\alpha^{2} \pm 2 q g\right)^{2}}+\left(\beta^{2}-q^{2}+g^{2}\right)}{2}$

$h^{2}=\frac{\sqrt{\left(\beta^{2}-q^{2}+g^{2}\right)^{2}+\left(\alpha^{2} \pm 2 q g\right)^{2}}-\left(\beta^{2}-q^{2}+g^{2}\right)}{2}$,

where $q>0, k>0$ always holds. The sign of $g$ or $h$ is determined accordingly (34). Alternatively, after having determined $q$ or $k$ from (35) and (37), $g$ or $h$ can be also determined directly from (34). Similar results as (35-38) are valid for $p, s$ with $g \rightarrow s, h \rightarrow p$.

The wavenumber $\kappa$ is simply determined by $\kappa=$ $\sqrt{q^{2}+k^{2}}=\sqrt{\beta^{2}+\varkappa^{2}+\psi^{2}}$, the angle $\theta$ giving the direction of propagation by $\tan \theta=k / q$.

If one sets in (23) $h=0$ ie if the wave amplitude is constant along the $z$ axis, then (35) and (36) yield

$$
\begin{gathered}
q^{2}=\frac{1}{2}\left\{\left(\beta^{2}-k^{2}\right)+\sqrt{\left(\beta^{2}-k^{2}\right)^{2}+\alpha^{4}}\right\}, \\
g^{2}=\frac{1}{2}\left\{-\left(\beta^{2}-k^{2}\right)+\sqrt{\left(\beta^{2}-k^{2}\right)^{2}+\alpha^{4}}\right\}, \\
q g=\mp \alpha^{2} / 2 .
\end{gathered}
$$

On the other hand, if one sets in (23) $g=0$ ie if the wave amplitude is constant along the $x$ axis, then (37) and (38) yield

$$
\begin{gathered}
k^{2}=\frac{1}{2}\left\{\left(\beta^{2}-q^{2}\right)+\sqrt{\left(\beta^{2}-q^{2}\right)^{2}+\alpha^{4}}\right\}, \\
h^{2}=\frac{1}{2}\left\{-\left(\beta^{2}-q^{2}\right)+\sqrt{\left(\beta^{2}-q^{2}\right)^{2}+\alpha^{4}}\right\}, \\
k h=\mp \alpha^{2} / 2 .
\end{gathered}
$$


In the limiting case of lossless ideal dieletrics, ie if $\alpha=0, \gamma=j \beta$ and if $k<\beta$ one gets classical result of obliquely propagating homogeneous plane wave

$$
\begin{aligned}
\mathbf{E}(\boldsymbol{r}) & =\boldsymbol{u}_{y} E \exp \left(-j \beta \mathbf{n}_{\ell} \cdot \boldsymbol{r}\right) \\
& =\boldsymbol{u}_{y} E \exp (-j \beta \zeta)=\mathbf{u}_{y} E \exp \{-j(q x+k z)\},
\end{aligned}
$$

with $g=h=0 \kappa=\beta$ (see (2), (3)), and $q=\beta \cos \theta$, $k=\beta \sin \theta, \beta^{2}=q^{2}+k^{2}$.

For $k=\beta$ one obtains $q=\alpha / \sqrt{2}$ and $g=\mp \alpha / \sqrt{2}$. In the limit $\alpha \rightarrow 0$ the result is $q=g=0$ and the propagating wave is a homogeneous plane wave propagating in direction of the $z$ axis $\mathbf{E}(\boldsymbol{r})=\boldsymbol{u}_{y} E \exp (-j k z)$.

If $k>\beta$ had been chosen, then from (39), (40) $q=$ $0, g= \pm \sqrt{k^{2}-\beta^{2}}$ and instead of (45), ie instead of a homogeneous wave propagating in direction of $\mathbf{n}_{\ell}$ one obtains an evanescent plane wave propagating in direction of the $z$ axis with positive, or negative evanescence in direction of the $x$ axis

$$
\mathbf{E}(\boldsymbol{r})=\mathbf{u}_{y} E \exp (g x) \exp (-j k z)
$$

This is in fact an expression identical with (11), for $g=\varkappa$ and $k=\kappa$.

\section{Propagation of evanescent plane waves along the planar boundary between various combinations of gainy, lossy, or lossless media and reflection and refraction effects}

Let us consider planar boundary $x=0$ between the two half spaces, $x<0$ and $x>0$ filled respectively with the lossy/gainy media having propagation constants

$$
\gamma_{1}=\sqrt{-\beta_{1}^{2} \pm j \alpha_{1}^{2}}, \quad \gamma_{2}=\sqrt{-\beta_{2}^{2} \pm j \alpha_{2}^{2}} .
$$

The complete solution of (5) for the overall wave $\mathbf{E}(\boldsymbol{r})=\mathbf{E}_{1,2}(\boldsymbol{r})=\mathbf{u}_{y} E_{1,2}(x, z)$ propagating (and being eventually attenuated, or amplified) in $z$ direction along the boundary $x=0$ consists of two TE waves $\mathbf{E}_{1}$ and $\mathbf{E}_{2}$ in each respective half space $x<0$ and $x>0$ in the form

$$
\mathbf{E}_{1,2}(x . z)=\boldsymbol{u}_{y} E_{1,2}(x) \exp \{(-j k+h) z\} .
$$

After having inserted (48) into (5) one obtains

$$
\begin{aligned}
& E_{1}(x)=E_{1}^{\text {in }} \exp \left\{\left(-j q_{1}+g_{1}\right) x\right\}+E_{1}^{\text {out }} \exp \left\{\left(j q_{1}-g_{1}\right) x\right\}, \\
& E_{2}(x)=E_{2}^{\text {in }} \exp \left\{\left(j q_{2}-g_{2}\right) x\right\}+E_{2}^{\text {out }} \exp \left\{\left(-j q_{2}+g_{2}\right) x\right\}
\end{aligned}
$$

The respective $q_{1,2}$ and $g_{1,2}$ are obtained from (34), (35) and (36) for respective $\beta_{1,2}, \alpha_{1,2}$ pertaining to the media parameters $\gamma_{1}$ and $\gamma_{2}$ with $k$ and $q_{1,2}$ always positive and the sign of $g_{1,2}$ determined by (34).

The above results can be represented in each half space as a superposition of two evanescent waves, given in form of (15), as illustrated in Fig. 1, yielding in the half space $x<0$

$$
\begin{aligned}
& \mathbf{E}_{1}(\boldsymbol{r})=\mathbf{E}_{1}^{\text {in }}(\boldsymbol{r})+\mathbf{E}_{1}^{\text {out }}(\boldsymbol{r})= \\
& \mathbf{u}_{y}\left[E_{1}^{\text {in }} \exp \left(\varkappa_{1} \boldsymbol{n}_{t 1}^{\text {in }} \cdot \boldsymbol{r}\right) \exp \left\{\left(-j \kappa_{1}+\psi_{1}\right) \boldsymbol{n}_{\ell 1}^{\text {in }} \cdot \boldsymbol{r}\right\}+\right. \\
& \left.E_{1}^{\text {out }} \exp \left(\varkappa_{1} \boldsymbol{n}_{t 1}^{\text {out }} \cdot \boldsymbol{r}\right) \exp \left\{\left(-j \kappa_{1}+\psi_{1}\right) \boldsymbol{n}_{\ell 1}^{\text {out }} \cdot \boldsymbol{r}\right\}\right],
\end{aligned}
$$

and in the half space $x>0$

$$
\begin{aligned}
& \mathbf{E}_{2}(\boldsymbol{r})=\mathbf{E}_{2}^{\text {in }}(\boldsymbol{r})+\mathbf{E}_{2}^{\text {out }}(\boldsymbol{r})= \\
& \mathbf{u}_{y}\left[E_{2}^{\text {in }} \exp \left(\varkappa_{2} \mathbf{n}_{t 2}^{\text {in }} \cdot \boldsymbol{r}\right) \exp \left\{\left(-j \kappa_{2}+\psi_{2}\right) \mathbf{n}_{\ell 2}^{\text {in }} \cdot \boldsymbol{r}\right\}+\right. \\
& \left.E_{2}^{\text {out }} \exp \left(\varkappa_{2} \mathbf{n}_{t 2}^{\text {out }} \cdot \boldsymbol{r}\right) \exp \left\{\left(-j \kappa_{2}+\psi_{2}\right) \boldsymbol{n}_{\ell 2}^{\text {out }} \cdot \boldsymbol{r}\right\}\right]
\end{aligned}
$$

where

$$
\begin{aligned}
& \kappa_{1}=\sqrt{q_{1}^{2}+k^{2}}=\sqrt{\beta_{1}^{2}+\varkappa_{1}^{2}+\psi_{1}^{2}}=\sqrt{\beta_{1}^{2}+g_{1}^{2}+h^{2}}, \\
& \kappa_{2}=\sqrt{q_{2}^{2}+k^{2}}=\sqrt{\beta_{2}^{2}+\varkappa_{2}^{2}+\psi_{2}^{2}}=\sqrt{\beta_{2}^{2}+g_{2}^{2}+h^{2}} .
\end{aligned}
$$

The angles $\theta_{1}, \theta_{2}$ are determined from $\sin \theta_{1}=k / \kappa_{1}$, $\sin \theta_{2}=k / \kappa_{2}$, thus defining all four propagation and transversal direction vectors $\boldsymbol{n}_{\ell} \quad \boldsymbol{n}_{t}$ given by (14) and (26). In fact they represent the standard Snellius law of reflection and refraction, modified for the lossy/gainy medium and positive/negative evanescence, since from $k=\kappa_{1} \sin \theta_{1}=\kappa_{2} \sin \theta_{2}$, directly stems, see (8),

$$
\sin \theta_{1} / \sin \theta_{2}=\kappa_{2} / \kappa_{1}
$$

Parameters $\varkappa_{1,2}$ and $\psi_{1,2}$ are determined from (22) for pertaining $\theta_{1,2}, g_{1,2}$ and $h$.

Each of the fields $\mathbf{E}_{1}$ and $\mathbf{E}_{2}$ in (51) and (52) (see Fig. 1) is given as a superposition of two partial evanescent plane waves, $\mathbf{E}_{1,2}^{\text {in }}$ propagating towards the boundary (inbound waves), and $\mathbf{E}_{1,2}^{\text {out }}$ propagating outwards the boundary (outbound waves). Propagation directions are thus given by (14), (26)

$$
\begin{aligned}
\boldsymbol{n}_{\ell 1}^{\text {in }} & =\boldsymbol{u}_{z} \sin \theta_{1}+\boldsymbol{u}_{x} \cos \theta_{1}, \\
\boldsymbol{n}_{\ell 1}^{\text {out }} & =\boldsymbol{u}_{z} \sin \theta_{1}-\boldsymbol{u}_{x} \cos \theta_{1}, \\
\mathbf{n}_{\ell 2}^{\text {in }} & =\boldsymbol{u}_{z} \sin \theta_{2}-\boldsymbol{u}_{x} \cos \theta_{2}, \\
\mathbf{n}_{\ell 2}^{\text {out }} & =\boldsymbol{u}_{z} \sin \theta_{2}+\mathbf{u}_{x} \cos \theta_{2},
\end{aligned}
$$

and $\boldsymbol{n}_{t}=\boldsymbol{u}_{y} \times \boldsymbol{n}_{\ell}$ holds for all $\boldsymbol{n}_{t}$.

The issue of a proper causal sequence has to be addressed. It is quite natural to consider the inbound waves in (51) and (52), coming from infinity and impinging on the boundary, as the primary independent entities - the waves out radiated from some sources in infinity. On the other hand the outbound waves are considered as secondary waves, ie as a causal consequence of interaction of primary inbound waves with the boundary. 

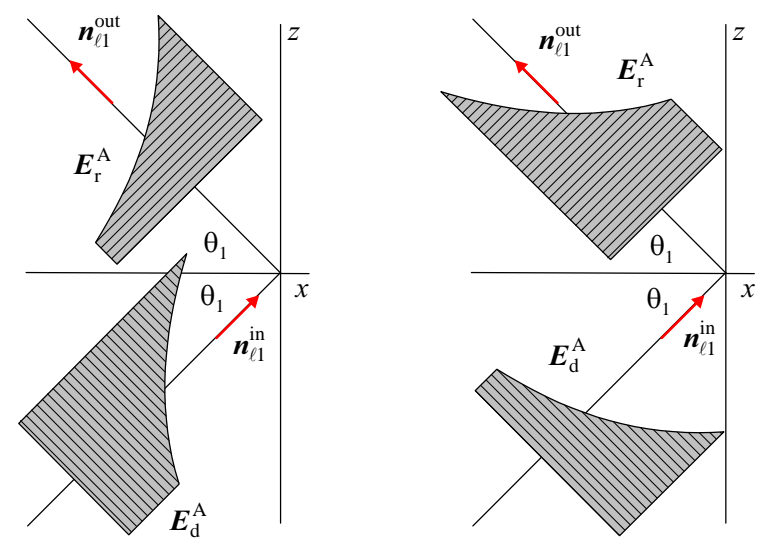

Fig. 2. Schematics of incident and reflected evanescent waves $\mathbf{E}_{d}^{A}$, $\mathbf{E}_{r}^{A}$ in attenuating media (left) - incident wave with positive evanescence, reflected wave with negative evanescence, and in amplifying media (right) - incident wave with negative evanescence, reflected wave with positive evanescence

On the boundary $x=0$ the wave amplitude must be continuous, ie the relation

$$
E_{1}^{\text {in }}+E_{1}^{\text {out }}=E_{2}^{\text {in }}+E_{2}^{\text {out }}
$$

must be fulfilled. The continuity condition of the tangential components of $\mathbf{H}_{1}$ and $\mathbf{H}_{2}$ vectors leads to the continuity of the $x$ derivatives of (49) and (50) on the boundary, thus yielding

$$
\left(q_{1}+j g_{1}\right)\left(E_{1}^{\text {in }}-E_{1}^{\text {out }}\right)=\left(q_{2}+j g_{2}\right)\left(E_{2}^{\text {out }}-E_{2}^{\text {in }}\right) .
$$

From (54) and (55) one may express any two of the $E$ quantities by the two others. It is logical to express outgoing waves $E_{1}^{\text {out }}$ and $E_{2}^{\text {out }}$ propagating outwards the boundary by the primary independent inbound waves $E_{1}^{\text {in }}$ and $E_{2}^{\text {in }}$ propagating from infinity towards the boundary. The result reads

$$
\begin{aligned}
& E_{1}^{\text {out }}=\rho E_{1}^{\text {in }}+(1-\rho) E_{2}^{\text {in }}, \\
& E_{2}^{\text {out }}=(1+\rho) E_{1}^{\text {in }}-\rho E_{2}^{\text {in }},
\end{aligned}
$$

where

$$
\rho=\frac{\left(q_{1}+j g_{1}\right)-\left(q_{2}+j g_{2}\right)}{\left(q_{1}+j g_{1}\right)+\left(q_{2}+j g_{2}\right)} .
$$

For example, the two parts of an overall wave propagating along the boundary $x=0$ for special case $E_{1}^{\text {in }}=E_{2}^{\text {in }}=E_{0}$ are

$$
\begin{aligned}
& \mathbf{E}_{1,2}(\boldsymbol{r})=\mathbf{u}_{y} 2 E_{0}\left[\cos \left(q_{1,2} x\right) \cosh \left(g_{1,2} x\right)\right. \\
&\left.-j \sin \left(q_{1,2} x\right) \sinh \left(g_{1,2} x\right)\right] \exp \{(-j k+h) z\} .
\end{aligned}
$$

The result is a non diffracting wave propagating (and being attenuated, or amplified) in $z$ direction, consisting in fact in each domain of superposition of the two non homogeneous plane waves, the first with the amplitude distribution $\cos \left(q_{1,2} x\right) \cosh \left(g_{1,2} x\right)$ and zero phase and the second with the amplitude distribution $\sin \left(q_{1,2} x\right) \sinh \left(g_{1,2} x\right)$ and constant phase $-\pi / 2$.
Recalling the standard textbook approach to reflection and refraction of obliquely incident homogeneous plane waves upon the planar boundary, it is easily seen that the above described configuration consists of two triples of incident, reflected and transmitted (refracted) waves (Fig. 1).

The first triple of partial configuration (denoted by upper index $A$ consists of primary incident wave with amplitude in origin $E_{1}^{\text {in }}=E_{d}^{A}$ obliquely impinging upon the boundary $x=0$ from negative $x$ infinity $(x \rightarrow-\infty)$ in domain $x<0$ (propagation constant $\gamma_{1}$

$$
\mathbf{E}_{d}^{A}(\boldsymbol{r})=\mathbf{u}_{y} E_{d}^{A} \exp \left\{\left(-j q_{1}+g_{1}\right) x\right\} \exp \{(-j k+h) z,
$$

further of the secondary reflected wave

$$
\mathbf{E}_{r}^{A}(\boldsymbol{r})=\mathbf{u}_{y} E_{r}^{A} \exp \left\{\left(j q_{1}-g_{1}\right) x\right\} \exp \{(-j k+h) z\},
$$

with amplitude in origin $E_{r}^{A}=\rho_{1} E_{d}^{A}$ together with the secondary transmitted (refracted) wave in domain $x>0$ (propagation constant $\gamma_{2}$ )

$$
\mathbf{E}_{p}^{A}(\boldsymbol{r})=\mathbf{u}_{y} E_{p}^{A} \exp \left\{\left(-j q_{2}+g_{2}\right) x\right\} \exp \{(-j k+h) z\},
$$

with amplitude in origin $E_{p}^{A}=\tau_{12} E_{d}^{A}$ where reflection coefficient $\rho_{1}=\rho$ and transmission coefficient $\tau_{12}=$ $1+\rho,(57)$. The difference in shape between incident and reflected evanescent waves in lossy versus gainy media is schematically illustrated in Fig. 2.

The second triple of partial configuration (denoted by upper index $B$ consists of primary incident wave with amplitude in origin $E_{2}^{\text {in }}=E_{d}^{B}$ obliquely impinging upon the boundary $x=0$ from positive $x$ infinity $(x \rightarrow \infty)$ in domain $x>0$ (propagation constant $\gamma_{2}$ )

$$
\mathbf{E}_{d}^{B}(\boldsymbol{r})=\mathbf{u}_{y} E_{d}^{B} \exp \left\{\left(j q_{2}-g_{2}\right) x\right\} \exp \{(-j k+h) z\},
$$

together with the secondary reflected and transmitted waves in respective domains, ie

$$
\mathbf{E}_{r}^{B}(\boldsymbol{r})=\boldsymbol{u}_{y} E_{r}^{B} \exp \left\{\left(-j q_{2}+g_{2}\right) x\right\} \exp \{(-j k+h) z\},
$$

$$
\mathbf{E}_{p}^{B}(\boldsymbol{r})=\boldsymbol{u}_{y} E_{p}^{B} \exp \left\{\left(j q_{1}-g_{1}\right) x\right\} \exp \{(-j k+h) z\},
$$

with amplitudes in origin $E_{r}^{B}=\rho_{2} E_{d}^{B}, E_{p}^{B}=\tau_{21} E_{d}^{B}$ where the reflection coefficient $\rho_{2}=-\rho$ and the transmission coefficient $\tau_{21}=1-\rho(57)$.

The primary incident waves $\mathbf{E}_{d}^{A}$ or $\mathbf{E}_{d}^{B}$ determine in each triple the independent parameters $q_{1}, k, g_{1}, h$ or $q_{2}, k, g_{2}, h$, respectively. The pertaining (always positive) dependent parameters $q_{2}$ or $q_{1}$ respectively, are determined from (35). with either $\beta_{2}, \alpha_{2}$ or $\beta_{1}, \alpha_{1}$ and $k, h$ together with $g_{1}, g_{2}$ determined from (34) as $g_{1,2}=\left(\mp \alpha_{1,2}^{2} / 2-k h\right) / q_{1,2}$. 


\section{Special case I: Reflection and refraction of a homogeneous plane wave}

Let us consider special case of an incident homogeneous plane wave $\mathbf{E}_{d}^{A}$ with zero evanescence, $\varkappa_{1}=0$ in domain $x<0$ impinging on the boundary, $x=0$ ie the incident and reflected wave (58), (59) take the form of attenuated/amplified homogeneous plane waves

$$
\begin{aligned}
\mathbf{E}_{d}^{A}(\boldsymbol{r}) & =\mathbf{u}_{y} E_{d}^{A} \exp \left\{-j\left(\kappa_{1}+j \psi_{1}\right) \boldsymbol{n}_{\ell 1}^{\text {in }} \cdot \boldsymbol{r}\right\} \\
= & \left.\mathbf{u}_{y} E_{d}^{A} \exp \left\{-j q_{1}+g_{1}\right) x\right\} \exp \{(-j k+h) z\}, \\
\mathbf{E}_{r}^{A}(\boldsymbol{r}) & =\mathbf{u}_{y} E_{r}^{A} \exp \left\{-j\left(\kappa_{1}+j \psi_{1}\right) \boldsymbol{n}_{\ell 1}^{\text {out }} \cdot \boldsymbol{r}\right\} \\
= & \left.\mathbf{u}_{y} E_{r}^{A} \exp \left\{-j q_{1}+g_{1}\right) x\right\} \exp \{(-j k+h) z\},
\end{aligned}
$$

where, see (9), (10),

$$
\begin{aligned}
& \kappa_{1}^{2}=\frac{1}{2}\left\{\beta_{1}^{2}+\sqrt{\beta_{1}^{4}+\alpha_{1}^{4}}\right\}, \\
& \psi_{1}^{2}=\frac{1}{2}\left\{-\beta_{1}^{2}+\sqrt{\beta_{1}^{4}+\alpha_{1}^{4}}\right\}, \\
& h=\vartheta_{1}=\psi_{1} \sin \theta_{1}, \quad g_{1}=\chi_{1}=\psi_{1} \cos \theta_{1}, \\
& k=\kappa_{1} \sin \theta_{1}, \quad q_{1}=\kappa_{1} \cos \theta_{1},
\end{aligned}
$$

both either attenuated for $\psi_{1}<0$ or amplified for $\psi_{1}>0$ depending on whether the medium with $\gamma_{1}$ is lossy or gainy.

The wave $\mathbf{E}_{p}^{A}(\boldsymbol{r})$ transmitted into the second domain $x>0$ ie into the medium with $\gamma_{2}$ is given as

$$
\mathbf{E}_{p}^{A}(\boldsymbol{r})=\mathbf{u}_{y} E_{p}^{A} \exp \left\{\left(-j q_{2}+g_{2}\right) x\right\} \exp \{(-j k+h) z\} .
$$

Parameter $q_{2}$ is determined from (35) for $\beta_{2}, \alpha_{2}, k$ and $h$. Then $\kappa_{2}=\sqrt{q_{2}^{2}+k^{2}}, \theta_{2}=\arcsin \left(k / \kappa_{2}\right)$, further $g_{2}=\left(\mp \alpha_{2}^{2}-2 k h\right) / 2 q_{2}$ and

$$
\begin{aligned}
& \varkappa_{2}=g_{2} \sin \theta_{2}-h \cos \theta_{2}, \\
& \psi_{2}=g_{2} \cos \theta_{2}+h \sin \theta_{2} .
\end{aligned}
$$

If both media, with $\gamma_{1}, \gamma_{2}$ are lossy, then in (64), (65) and (66) $\psi_{1}, \psi_{2}<0$, if gainy then $\psi_{1}, \psi_{2}>0$. If it happened that $h=\psi_{1} \sin \theta_{1}=\psi_{2} \sin \theta_{2}$ then the evanescence factor $\varkappa_{2}$ is zero too. In such a case the wave $\mathbf{E}_{p}^{A}(\boldsymbol{r})$ propagating in the second medium were a homogeneous plane wave too, being just attenuated (or amplified) in direction of propagation as $\exp \left(\psi_{2} \boldsymbol{n}_{\ell 2}^{\text {out }} \cdot \boldsymbol{r}\right)$

If both media are gainy and $\psi_{1} \sin \theta_{1}>\psi_{2} \sin \theta_{2}$ then the growth along the boundary $x=0$ must be supported in media $\gamma_{2}$ by a positive evanescence $\varkappa_{2}<0$. If $\psi_{1} \sin \theta_{1}<\psi_{2} \sin \theta_{2}$ then the growth along the boundary must, in media with $\gamma_{2}$, be suppressed by a negative evanescence $\varkappa_{2}>0$, in order to reach in both cases $\psi_{2} \sin \theta_{2}-\varkappa_{2} \cos \theta_{2}=\psi_{1} \sin \theta_{1}$.

If both media are lossy and $\psi_{2} \sin \theta_{2}<\psi_{1} \sin \theta_{1}$, $\psi_{1,2}<0$ then the decay along the boundary $x=0$ must be supported in media $\gamma_{2}$ by a negative evanescence $\varkappa_{2}>0$. If, vice versa, $\psi_{2} \sin \theta_{2}>\psi_{1} \sin \theta_{1}$ then the decay along the boundary must be suppressed in media $\gamma_{2}$ by a positive evanescence $\varkappa_{2}<0$ in order, in both cases, to reach $\psi_{2} \sin \theta_{2}-\varkappa_{2} \cos \theta_{2}=\psi_{1} \sin \theta_{1}$.

For the case of a lossless medium in domain $x>0$, $\gamma_{2}=j \beta_{2}=j \omega \sqrt{\mu \varepsilon_{2}}, \alpha_{2}=0, \psi_{2}=0$, the whole decay, or growth, $\exp (h z)$ along the $z$ axis, must be carried out by either the negative, or the positive evanescence of propagating undamped plane wave in domain $x>0$ ie $h=-a=-\varkappa_{2} \cos \theta_{2}$ with pertaining $g_{2}=b_{2}=$ $\varkappa_{2} \sin \theta_{2}$

The incident and reflected wave remain as given by (64) and (65) but $\mathbf{E}_{p}^{A}(\mathbf{r})$ is now given by

$$
\begin{gathered}
\mathbf{E}_{p}^{A}(\boldsymbol{r})=\boldsymbol{u}_{y} E_{p}^{A} \exp \left(\varkappa_{2} \boldsymbol{n}_{t 2}^{\text {out }} \cdot \boldsymbol{r}\right) \exp \left\{-j \kappa_{2} \boldsymbol{n}_{\ell 2}^{\text {out }} \cdot \boldsymbol{r}\right\} \\
\quad=\boldsymbol{u}_{y} E_{p}^{A} \exp \left\{\left(-j q_{2}+g_{2}\right) x\right\} \exp \{(-j k+h) z\},
\end{gathered}
$$

where, see (35),

$$
q_{2}^{2}=\frac{1}{2}\left\{\sqrt{\left(\beta_{2}^{2}-k^{2}+h^{2}\right)^{2}+(2 k h)^{2}}+\left(\beta_{2}^{2}-k^{2}+h^{2}\right)\right\},
$$

$q_{2}$ having always positive value, and $g_{2}$ determined from $g_{2}=-k h / q_{2}$ leading to, if $h>0, g_{2}<0$ and vice versa, if $h<0, g_{2}>0$.

In other words, if $h<0$ (lossy medium in $x<0$ ), ie $\exp (h z)$ is decreasing for $z \rightarrow \infty$, then $\exp \left(g_{2} x\right)$ is exponentially growing to infinity with growing distance $x$ from the boundary. On the other hand, if $h>0$ (gainy medium in $x<0)$, ie $\exp (h z)$ is increasing for $z \rightarrow \infty$ then $\exp \left(g_{2} x\right)$ converges to zero with growing distance $x$ from the boundary.

\section{Special case II: Boundary between lossless and lossy/gainy medium with homogeneous plane wave in lossless medium}

Let us now consider the complementary case of lossless dielectrics in domain $x<0$ ie $\gamma_{1}=j \beta_{1}=j \omega \sqrt{\mu \varepsilon_{1}}$ and lossy/gainy nedium in domain $x>0$. Then $\psi_{1}=0$ and instead of (58), (59) we have to deal with undamped homogeneous incident and reflected waves in domain $x<$ 0 ie

$$
\begin{aligned}
& \mathbf{E}_{d}^{A}(\mathbf{r})=\mathbf{u}_{y} E_{d}^{A} \exp \left\{-j \beta_{1} \boldsymbol{n}_{\ell 1}^{\text {in }} \cdot \boldsymbol{r}\right\} \\
& =\mathbf{u}_{y} E_{d}^{A} \exp \left(-j q_{1} x\right) \exp (-j k z), \\
& \begin{aligned}
\mathbf{E}_{r}^{A}(\mathbf{r})=\mathbf{u}_{y} E_{r}^{A} \exp & \left\{-j \beta_{1} \boldsymbol{n}_{\ell 1}^{\text {out }} \cdot \boldsymbol{r}\right\} \\
& =\mathbf{u}_{y} E_{r}^{A} \exp \left(j q_{1} x\right) \exp (-j k z),
\end{aligned}
\end{aligned}
$$

where $q_{1}=\beta_{1} \cos \theta_{1}, k=\beta_{1} \sin \theta_{1}$. The wave amplitude $\mathbf{E}_{1}=\mathbf{E}_{d}^{A}+\left.\mathbf{E}_{r}^{A}\right|_{x=0}$ on the boundary $x=0$ is constant, ie $h=0$. Therefore $h$ in (60) must be zero too and

$$
\begin{array}{r}
\mathbf{E}_{p}^{A}(\boldsymbol{r})=\mathbf{u}_{y} E_{p}^{A} \exp \left(\varkappa_{2} \boldsymbol{n}_{t 2}^{\text {out }} \cdot \boldsymbol{r}\right) \exp \left\{-j\left(\kappa_{2}+j \psi_{2}\right) \mathbf{n}_{\ell 2}^{\text {out }} \cdot \boldsymbol{r}\right\} \\
=\mathbf{u}_{y} E_{p}^{A} \exp \left\{\left(-j q_{2}+g_{2}\right) x\right\} \exp (-j k z),
\end{array}
$$




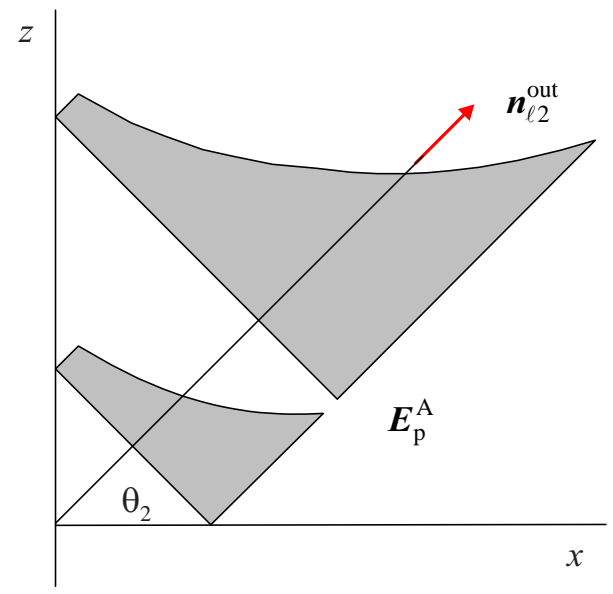

Fig. 3. Schematics of the refracted evanescent wave in an amplifying medium with constant amplitude on the boundary.

where $q_{2}$ is determined from (39) for $\beta_{2}$ and $\alpha_{2}$

$$
q_{2}^{2}=\frac{1}{2}\left\{\left(\beta_{2}^{2}-k^{2}\right)+\sqrt{\left(\beta_{2}^{2}-k^{2}\right)^{2}+\alpha_{2}^{4}}\right\}
$$

and always takes positive value, while $g_{2}$ is determined from (41)as $g_{2}=\mp \alpha_{2}^{2} / 2 q_{2}$, ie $g_{2}<0$ for the lossy second medium and $g_{2}>0$ for the gainy one.

In order to have the wave $\mathbf{E}_{p}^{A}$ on the boundary $x=0$ constant, the decay, or growth of wave amplitude, due to lossy/gainy medium in domain $x>0$ must be compensated by its evanescence in such a way that $\psi_{2} \sin \theta_{2}-$ $\varkappa_{2} \cos \theta_{2}=0$ ie for lossy medium $\left(\psi_{2}<0\right)$ by positive evanescence $\varkappa_{2}<0$ and for gainy medium $\left(\psi_{2}>0\right)$ by negative one $\varkappa_{2}>0$. Therefore in lossy medium the wave amplitude is in $x$ direction attenuated, $g_{2}<0$, while in gainy medium amplified, $g_{2}>0$, where $g_{2}=\psi_{2} / \cos \theta_{2}$. An example of the evanescent plane wave in amplifying medium with the constant wave amplitude along the $z$ axis is illustrated in Fig. 3.

\section{The ambiguity in transition from lossy/gainy media to two lossless media}

The transition to the case of two lossless media, ie to $\alpha_{1,2}=0$ from either when the medium in domain $x<0$ is lossy or gainy, $\alpha_{1} \neq 0$, and medium in domain $x>0$ is lossless, $\alpha_{2}=0$ (Special case I), or, vice versa, when the medium in $x<0$ is lossless, $\alpha_{1}=0$ and medium in $x>0$ is lossy or gainy, $\alpha_{2} \neq 0$ (Special case II), in other words, the limiting case when either $\alpha_{1}$ in Special case I, or $\alpha_{2}$ in Special case II, converges to zero is worth mentioning.

The two distinct cases of two lossless media must be recognized. The first case when $\beta_{2}>\beta_{1}$ ie when $\varepsilon_{2}>\varepsilon_{1}$, $\theta_{2}<\theta_{1}$ ie when the transmission from the so called optically thinner into optically denser medium occurs, and the second case, when $\beta_{1}>\beta_{2}$ ie when $\varepsilon_{1}>\varepsilon_{2}$, $\theta_{1}<\theta_{2}$ ie when the transmission from the so called optically denser into optically thinner medium occurs.
In the first case, $\beta_{2}>\beta_{1}>k, \theta_{1}>\theta_{2}, k=\beta_{1} \sin \theta_{1}=$ $\beta_{2} \sin \theta_{2}$ always hold. For Special case I, when the second media is lossless and the first media converges from loosy/gainy to lossless, $i e$ to the limit $\alpha_{1} \rightarrow 0$ the following parameters in (64), (65) converge to theirs limits $\psi_{1} \rightarrow 0, \kappa_{1} \rightarrow \beta_{1}, h \rightarrow 0, g_{1} \rightarrow 0, q_{1}=\beta_{1} \cos \theta_{1}$ and $\mathbf{E}_{d}^{A}(\boldsymbol{r}), \mathbf{E}_{r}^{A}(\boldsymbol{r})$ in (64), (65) become standard homogeneous plane waves

$$
\begin{aligned}
\begin{aligned}
\mathbf{E}_{d}^{A}(\boldsymbol{r})=\mathbf{u}_{y} E_{d}^{A} \exp \{ & \left.-j \beta_{1} \boldsymbol{n}_{\ell 1}^{\text {in }} \cdot \boldsymbol{r}\right\} \\
& =\mathbf{u}_{y} E_{d}^{A} \exp \left\{-j\left(q_{1} x+k z\right)\right\}, \\
\mathbf{E}_{r}^{A}(\boldsymbol{r})=\boldsymbol{u}_{y} E_{r}^{A} \exp \{- & \left.j \beta_{1} \boldsymbol{n}_{\ell 1}^{\text {out }} \cdot \boldsymbol{r}\right\} \\
& =\mathbf{u}_{y} E_{r}^{A} \exp \left\{j\left(q_{1} x-k z\right)\right\},
\end{aligned}
\end{aligned}
$$

with $E_{r}^{A}=\rho_{1} E_{d}^{A}, \rho_{1}=\left(q_{1}-q_{2}\right) /\left(q_{1}+q_{2}\right)$.

Since $h \rightarrow 0, g_{2} \rightarrow 0$ the transmitted wave $\mathbf{E}_{p}^{A}(\mathbf{r})$ in (68) becomes a standard homogeneous plane wave too

$$
\begin{aligned}
\mathbf{E}_{p}^{A}(\boldsymbol{r})=\boldsymbol{u}_{y} E_{p}^{A} \exp \{ & \left.-j \beta_{2} \boldsymbol{n}_{\ell 2}^{\text {out }} \cdot \boldsymbol{r}\right\} \\
& =\boldsymbol{u}_{y} E_{p}^{A} \exp \left\{-j\left(q_{2} x+k z\right)\right\},
\end{aligned}
$$

with $q_{2}=\beta_{2} \cos \theta_{2}, E_{p}^{A}=\tau_{12} E_{d}^{A}, \tau_{12}=2 q_{1} /\left(q_{1}+q_{2}\right)$.

When, vice versa, $\beta_{1}>\beta_{2}$ ie when $\varepsilon_{1}>\varepsilon_{2}$ then the two distinct cases again have to be recognized. The first case, when $\beta_{1}>\beta_{2}>k$ ie $k=\beta_{1} \sin \theta_{1}<\beta_{2}$ or $\theta_{1}<\theta_{c}$, where $\theta_{c}=\arcsin \left(\beta_{2} / \beta_{1}\right)$ is the critical angle, and the second case, when $\beta_{1}>k>\beta_{2}$, ie $k=\beta_{1} \sin \theta_{1}>\beta_{2}$ or $\theta_{1}>\theta_{c}$ holds.

The second case is the case of the total internal reflection occurring when the angle of incident wave $\theta_{1}$ exceeds the critical angle $\theta_{c}=\arcsin \left(\beta_{2} / \beta_{1}\right)$.

In the case $k>\beta_{2}$, (69) can be written also as $q_{2}^{2}=\frac{1}{2}\left\{\sqrt{\left(k^{2}-\beta_{2}^{2}-h^{2}\right)^{2}+(2 k h)^{2}}-\left(k^{2}-\beta_{2}^{2}-h^{2}\right)\right\}$ leading for $h \rightarrow 0$ to $q_{2} \rightarrow 0$. Since $g_{2}$ now equals $g_{2}^{2}=\frac{1}{2}\left\{\sqrt{\left(k^{2}-\beta_{2}^{2}-h^{2}\right)^{2}+(2 k h)^{2}}+\left(k^{2}-\beta_{2}^{2}-h^{2}\right)\right\}$, the limit $h \rightarrow 0$ yields $g_{2}^{2}=k^{2}-\beta_{2}^{2}$. Since $q_{2}$ is always non negative and $g_{2}=-k h / q_{2}$ in the limit $h \rightarrow 0$ one obtains $g_{2}=\sqrt{k^{2}-\beta_{2}^{2}}$ for $h$ converging to zero from negative values, ie converging to lossless case from lossy medium, while for for $h$ converging to zero from positive values, ie, converging to lossless case from gainy medium, one obtains $g_{2}=-\sqrt{k^{2}-\beta_{2}^{2}}$.

The incident and reflected wave take the form of standard homogeneous plane waves as in (74), (75) too, but with $\rho_{1}=\left(q_{1}-j g_{2}\right) /\left(q_{1}+j g_{2}\right)=\exp (j \Psi)$, ie $\left|\rho_{1}\right|=1$. Thus one obtains

$$
\mathbf{E}_{p}^{A}(\boldsymbol{r})=\mathbf{u}_{y} E_{p}^{A} \exp \left(g_{2} x\right) \exp (-j k z)
$$

$i e$ an evanescent wave propagating in direction of $z$ axis with propagation constant $k=\beta_{1} \sin \theta_{1}$ with the amplitude exponentially increasing, or decreasing in direction 
of the $x$ axis, depending on whether the lossless case has been attained for convergence from lossy or gainy case.

Principally, the same results will be obtained for the case of total internal reflection by analogous procedure for the Special case II, $i e$ for the combination of lossless media in domain $x<0$ and lossy or gainy media in domain $x>0$ converging to two lossless media in both domains, with the difference that the signs in $g_{2}$ will be interchanged, ie $g_{2}=\sqrt{k^{2}-\beta_{2}^{2}}$ for convergence from gainy media, and $g_{2}=-\sqrt{k^{2}-\beta_{2}^{2}}$ for convergence from lossy media.

The controversy consists in the fact that in case of total internal reflection the result of convergence to the same final arrangement of two lossless media depends on whether the convergence starts in Special case I from lossy or gainy first medium (in domain $x<0$ ) and in Special case II from gainy or lossy second medium (in domain $x>0$ ). When starting from lossy or gainy first medium and lossless second medium the convergence to two lossless media leads to $g_{2}=\sqrt{k^{2}-\beta_{2}^{2}}$ for initially lossy first medium and $g_{2}=-\sqrt{k^{2}-\beta_{2}^{2}}$ for initially gainy first medum. Vice versa, when starting from lossy or gainy second medium and lossless first medium the convergence to two lossless media leads to $g_{2}=\sqrt{k^{2}-\beta_{2}^{2}}$ for initially gainy second medium and $g_{2}=-\sqrt{k^{2}-\beta_{2}^{2}}$ for initially lossy second medum.

However, it is worth to mention, that when starting $a b$ initio from two lossless media and condition of total internal reflection $k>\beta_{2}$ is fulfilled, then both solutions $g_{2}=\mp \sqrt{k^{2}-\beta_{2}^{2}}$ are mathematically correct. However only the solution converging to zero for $x \rightarrow \infty$ is considered to be physically eligible, therefore in (77) $g_{2}>0$ is generally rejected, and only $g_{2}=-\sqrt{k^{2}-\beta_{2}^{2}}$ is permissible.

\section{Resolving the ambiguity in transition from lossy/gainy media to two lossless media}

Some time ago the above mentioned ambiguity has been widely discussed in literature, see eg [4-7], in connection with the so-called "enhanced total internal reflection" within the framework of so called active optical (dielectric) waveguides - dielectric waveguides having lossless core and gainy cladding (due to excitation by lasing effects from the pump signal) and operating in regime of total internal reflection. The modes in such active waveguides are amplified along the propagation path. Expressed in our terms, this is our Special case II with the gainy second medium in domain $x>0$ When the pump signal is switched off, ie for lossless cladding (lossless second medium in our terms), the waveguide should operate in the standard regime. In our terms it means that $g_{2}$ in $\exp \left(g_{2} x\right)$ in $\mathbf{E}_{p}^{A}(\mathbf{r})$ in (77) must be negative. Paradoxically for Special case II and gainy medium in second domain $x>0$, ie for $\mathbf{E}_{p}^{A}(\mathbf{r})$ in (72) the lossless limit $\psi_{2} \rightarrow 0, \varkappa_{2} \rightarrow 0$ leads, in total internal reflection regime, to $g_{2}>0$.
To remedy the situation some authors in [4-7] maintained that when the angle of incident wave reaches and exceeds the critical angle $\theta_{1}=\theta_{c}$ then the direction of propagation of refracted wave $\boldsymbol{n}_{\ell 2}^{\text {out }}$ should be taken in opposite $x$ direction (ie towards the boundary), ie $\mathbf{E}_{p}^{A}(\boldsymbol{r})$ should propagate in $\boldsymbol{n}_{\ell 2}^{\text {in }}$ direction instead of $\boldsymbol{n}_{\ell 2}^{\text {out }}$. In fact it means to take instead of $\mathbf{E}_{p}^{A}(\boldsymbol{r})=\mathbf{E}_{2}^{\text {out }}(\boldsymbol{r})$ the wave $\mathbf{E}_{p}^{A}(\boldsymbol{r})=\mathbf{E}_{2}^{\text {in }}(\boldsymbol{r})$ as given in (52). Then the reflection coefficient $\rho_{1}=\left(q_{1}+q_{2}+j g_{2}\right) /\left(q_{1}-q_{2}-j g_{2}\right)$ indeed will have magnitude larger than one, $\left|\rho_{1}\right|>1$ the reflected wave the amplitude larger than the incident wave, ie amplified ("enhanced") total reflection occurs and the amplification of reflected wave takes place. Unfortunately, as shown in [8] such reversal of direction of propagation of the refracted wave cannot be physically justified and in fact there is not any reason for it to occur. As discussed above $\mathbf{E}_{2}^{\text {in }}(\boldsymbol{r})$ coming from plus infinity must be independent source quantity and not a result of any refraction on the boundary $x=0$.

As shown in [9] if one takes into account a second boundary in the half space $x>0$ in some distance $a$ from the first boundary, ie second boundary in plane $x=a$ then in the slab $0<x<a$ the previous wave $\mathbf{E}_{2}^{\text {out }}(\boldsymbol{r})$ transmitted from the domain $x<0$ through the first boundary, as well as the wave $\mathbf{E}_{2}^{\text {in }}(\boldsymbol{r})$ reflected on the second boundary, exist. The ratio between $\mathbf{E}_{2}^{\text {out }}(\boldsymbol{r})$ and $\mathbf{E}_{2}^{\text {in }}(\boldsymbol{r})$ depends on the properties of medium in the slab. If now $a$ converges to infinity $a \rightarrow \infty$, ie the second boundary is shifted to infinite distance from the first boundary, then in gainy medium indeed $\mathbf{E}_{2}^{\text {out }}$ converges to zero and only finite "reflected" wave $\mathbf{E}_{2}^{\text {in }}$ remains.

Another way how to circumvent this ambiguity is to use a somehow artificial two step limiting process. We describe it in some detail for Special case II.

In the first step $k=\beta_{1} \sin \theta_{1}<\beta_{2}$ converges to $\beta_{2}$ $k \rightarrow \beta_{2}$, ie increasing the incidence angle $\theta_{1}$ from $\theta_{1}<\theta_{c}$ to the critical angle $\theta_{1}=\theta_{c}$, leads to $q_{2}=\alpha_{2} / \sqrt{2}$, $g_{2}=\mp \alpha_{2} / \sqrt{2}$ see (39), (40), (41). Limiting now loss or gain in the second medium to zero, $\alpha_{2} \rightarrow 0$ one arrives to $q_{2}=g_{2}=0$ ie to the case of common critical angle incidence of a homogeneous plane wave on the boundary of two lossless dielectrics, with $\rho_{1}=1$ and $\tau_{12}=2$ resulting in a homogeneous plane wave propagating in domain $x>0$ along the boundary in $z$ direction

$$
\mathbf{E}_{p}^{A}(\mathbf{r})=\mathbf{u}_{y} 2 E_{d}^{A} \exp (-j k z)
$$

If now the angle of incidence is increased beyond the critical angle, ie $\theta_{1}>\theta_{c}, k$ becomes larger than $\beta_{2}, k=$ $\beta_{1} \sin \theta_{1}>\beta_{2}$ and $q_{2}=\sqrt{\beta_{2}^{2}-k^{2}}$ becomes purely imaginary with two roots $q_{2}=\mp j g_{2}=\mp j \sqrt{k^{2}-\beta_{2}^{2}}$ leading thus to $\exp \left(-j q_{2} x\right)=\exp \left(\mp g_{2} x\right)$, ie to the evanescent wave propagating along the boundary

$$
\mathbf{E}_{p}^{A}(\boldsymbol{r})=\boldsymbol{u}_{y} 2 E_{d}^{A} \cos (\Psi) \exp (j \Psi) \exp \left(\mp g_{2} x\right) \exp (-j k z)
$$


Both solutions are mathematically correct. Usually, reasoning by physical arguments only the exponentially decreasing solution $\exp \left(-g_{2} x\right)$ is considered eligible and taken into account.

\section{Conclusions}

Here we have thoroughly analysed the reflection and refraction of evanescent plane waves on the planar boundary between the various combinations of lossy, gainy, and lossless media and pointed out some up-to-now rarely discussed features of the solution. Of course, there is always the question connected with amplitude divergence (grow to infinity) of evanescent waves in an infinite half space and of theirs physical feasibility. The same question however, may be always connected with the infinite extension of commonly used homogeneous plane wave itself. They both must be always understood as theoretical concepts used to obtain useful physical insight into various facets of the theory. Practical problems are always solved in finite part of space and with spatially bounded waves.

\section{Acknowledgments}

This work has been supported by the Slovak Research and Development Agency under the contract no. APVV16-0059 and by the Scientific Grant Agency of the Ministry of Education, Science, Research and Sport of the Slovak Republic and in part by the Slovak Academy of Sciences, under project no. 1/0135/20.

\section{REFERENCES}

[1] N. Tralli, Classical Electromagnetic Theory, McGraw Hill 1963, New York.

[2] W. K. Panofsky and M. Philips, Classical Electricity and Magnetism, Addison Wesley 1955, Cambridge.

[3] J. D. Jackson, Classical Electrodynamics, Wiley 1962, New York.

[4] G. N. Romanov and S. S. Shakhidzanov, "Amplification of Electromagnetics Field Total Internal Reflection from a Region of Inverted Population", JETP Lett., vol. 16, no. 5, pp. 209-211, 1972.

[5] A. A. Kolokolov, "Reflection of Plane Waves from an Amplifying Medium", JETP Lett., vol. 21, no. 11, pp. 312-313, 1975.
[6] P. R. Callary and C. K. Carniglia, "Internal Reflection from an Amplifying Layer", Opt. Soc. Am., vol. 66, no. 8, pp. 775-779, 1976.

[7] W. Lukosz and P. P. Herrmann, "Amplification by Reflection from an Active Medium", Opt. Comm., vol. 17, no. 2, pp. 192-195, 1976.

[8] A. Siegman, "Fresnel Reflection, Lenserf Reflection and Evanescent Gain", Opt. and Photon. News, pp. 38-45, 2010.

[9] L'. Šumichrast, J. Franek,R. Dosoudil, and J. Červeňová, "Some Remarks on Enhanced Total Internal Reflection", Journal of Electrical Engineering, vol. 71, no. 1. pp. 20-30, 2020.

Received 23 July 2020

Jozefa Červenňová (Doc, Ing, PhD), born in Bratislava, graduated from the Faculty of Electrical Engineering and Information Technology of the Slovak University of Technology in Bratislava in 1971. She completed her PhD studies in 1993 in the eld of Electromagnetic Theory. Her current research interests concern mainly the testing methods of optical ber systems and components.

Rastislav Dosoudil (Doc, Ing, PhD) was born in Bratislava, in 1970. He graduated from Faculty of Electrical Engineering and Information Technology, Slovak University of Technology, in Bratislava, in Material Engineering branch, 1993 (technology of electronic equipments) and received the $\mathrm{PhD}$ degree in Theory of Electromagnetism in 2000. At present, he is an Associate Professor (2008) with the Institute of Electrical Engineering. He teaches subjects concerning electric circuits, electromagnetics and modern materials for electrical engineering. His research activities are mainly electromagnetic composite materials, high-frequency complex permittivity/permeability phenomena, electromagnetic wave absorbing properties of composites, and magnetic measurements.

Jaroslav Franek (Ing, CSc), graduated from the Faculty of Electrical Engineering, Slovak Technical University, Bratislava, in 1969, and received the CSc ( $\mathrm{PhD}$ ) degree in Physics in 1986. At present he is an emeritus Assistant Professor at the same Faculty. The main elds of his research are circuit and electromagnetic eld theory, namely the microwave technology.

L’ubomír Šumichrast (Doc, Ing, PhD) is an emeritus Associate Professor with the Faculty of Electrical Engineering and Information Technology of the Slovak University of Technology. His main research interests include the electromagnetic waves propagation in various media and structures, computer modelling of wave propagation eects as well as optical communication and integrated optics. 